\title{
Adverse health effects among nurses and clinical pharmacists handling antineoplastic drugs: Adherence to exposure control methods \\ Original
} Article

\author{
Noha S. Elshaer \\ Industrial Medicine and Occupational Health, Department of Community Medicine \\ Department, Faculty of Medicine, Alexandria University, Alexandria, Egypt
}

\begin{abstract}
Background: Chronic exposure to antineoplastic drugs (ADs) may result in reproductive, liver, renal, lung, and cardiac toxicity. Moreover, bone marrow suppression, mucosal ulcers, and cancer may develop. In developing countries, adverse health effects owing to occupational exposure to ADs and adherence to safe handling guidelines are not well documented. Aim: This study was conducted to determine the health effect of occupational exposure to ADs and evaluate adherence to control methods.

Materials and Methods: A comparative cross-sectional approach was adopted. ADs-exposed nurses and clinical pharmacists $(n=54)$ were compared with nonexposed group $(n=54)$. Self-reported clinical manifestations. and use of exposure controls were reported via an interview questionnaire. Blood samples were collected for complete blood count and liver and kidney function tests.

Results: Significantly higher rate of impaired fertility (31\%) and oral ulcers $(36.36 \%)$ were reported by ADs-exposed nurses and clinical pharmacists compared with nonexposed group (3.8 and 7.4\%, respectively; $P=0.01$ and $P=0.00$, respectively). Moreover, ADs-exposed group had significantly lower mean white blood cell count $(6518 \pm 2064.79 / \mu 1)$ and significantly higher mean creatinine level $(056 \pm 0.13 \mathrm{mg} / \mathrm{dl})$ compared with nonexposed group $(7307 \pm 2001.4 / \mu \mathrm{l}$ and $0.51 \pm 0.12 \mathrm{mg} / \mathrm{dl}$, respectively; $\mathrm{t}=2.02, P=0.04$; and $P=0.04$, respectively). Inadequate controls were reported, mainly lack of medical surveillance (100\%), lack of training (69.1\%), insufficient handling practices, and low usage of personal protective equipment, particularly among nurses.

Conclusion: The study highlighted chronic adverse effects associated with occupational exposure to ADs and inadequate implementation of exposure control methods. Findings necessitate raising awareness among ADs-exposed nurses and clinical pharmacists to introduce engineering controls, conduct hazard awareness training, initiate medical surveillance program, and ensure adherence to safe handling practices.
\end{abstract}

Received: 13 July 2017, Accepted: 07 Aug 2017

Key Words: Adverse effects, antineoplastic agents, clinical pharmacists, hazard control, nurses.

Corresponding Author: Noha S. Elshaer, M.D., Industrial Medicine and Occupational Health, Department of Community Medicine, Faculty of Medicine, Alexandria University, Alexandria, Egypt, Tel.: 00201005641972, E-mail: noha.alshaaer@ alexmed.edu.eg, elshaer.n@gmail.com

ISSN: 0013-2446, Vol. 92, No.3

\section{INTRODUCTION}

Antineoplastic agents (cancer chemotherapy drugs and cytotoxic drugs) affect both neoplastic cells as well as normal cells, particularly those cells that exhibit rapid activity and growth, such as bone marrow tissue and hair follicles ${ }^{[1]}$. Clinical pharmacists who prepare these drugs or nurses who administer them to patients (and/or sometimes prepare them) are the two occupational groups who have the highest potential exposure to antineoplastic drugs $(\mathrm{ADs})^{[2]}$. External contamination of vials by ADs residues and consequently contamination of air and surfaces in the pharmacy and administration work areas may reach more than $60 \%{ }^{[3]}$. Consequently, significant amounts of harmful agents can be absorbed via unprotected skin or inhalation of the powder and liquid aerosols $s^{[4,5]}$.
In literature, adverse health effects owing to longterm exposure to ADs in patients with cancer are welldocumented $^{[2]}$; however, most studies on occupational exposure to ADs have focused on environmental contamination or measurement of the exposure leve ${ }^{[4,6-9]}$. Studies that examined adverse health effects owing to occupational exposure to ADs were conducted in developed countries ${ }^{[4,7,8,10,11]}$. On the contrary, few studies demonstrated the health effect in developing countries such as China ${ }^{[12]}$. In Egypt, few studies were conducted 20 years ago to evaluate genotoxicity and chromosomal aberrations owing to occupational exposure to cytotoxic drugs ${ }^{[13,14]}$, and only one study was recently published ${ }^{[15]}$.

Studies revealed that reproductive toxicity may 
be presented in the form of infertility (temporary or permanent), spontaneous abortions, and preterm births ${ }^{[16,17]}$. In addition, organ toxicity such as liver, renal, lung, and cardiac toxicity may develop. Moreover, bone marrow suppression, mucosal ulcers, and bleeding could occur. Exposure to ADs might also lead to hair loss and infections owing to immunological suppression and reduced white blood cell (WBC) count ${ }^{[12]}$. Additionally, there is a risk of development of cancer ${ }^{[18]}$. The International Agency for Research on Cancer in Lyon, France, currently lists 11 antineoplastic agents and two combined therapies as group 1 (human carcinogens), 12 as group 2A (probable human carcinogens), and 11 as group $2 \mathrm{~B}$ (possible human carcinogens) $)^{[19]}$.

Factors that may increase the risk of contamination and occupational exposure include insufficient protective conditions at workplace, incorrect operation, lack of hazard awareness, unsafe work practices, lack of adherence to the use of personal protective equipment (PPE), and long-term occupational exposure ${ }^{[5,6,20]}$

Guidelines that include specific methods for the safe handling of ADs to alleviate workplace hazards have been available for approximately three decades ${ }^{[2,21-23]}$. Several studies in developed countries evaluated ADs exposure control methods including engineering, administrative, work practices controls, and PPE usage by ADs-exposed personnel ${ }^{[24-29]}$. Yet, in developing countries, information about adherence to exposure control methods that protect ADs-exposed healthcare workers during the performance of their jobs is not well documented ${ }^{[12]}$.

In Alexandria, Egypt, for a long period of time in the past years, the preparation and administration of injectable ADs in hospitals and oncology centers, have been handled by nurses in an open-plan treatment area under poorly controlled conditions. Recently in some hospitals and oncology centers, a centralized ADs preparation and admixture unit (CAPU) with better protection and safety precaution has been implemented to minimize contamination and occupational exposure to ADs. In CAPU, well-trained clinical pharmacists prepare, admix, and pack ADs inside biological safety cabinets, and then packed ADs are delivered to be administrated to patients by nurses in the treatment area. Although CAPU represents a safe way for reducing the potential occupational risk from ADs exposure ${ }^{[12]}$, yet few CAPUs exit in Alexandria with few number of clinical pharmacists (Elbadry I.M., Professor of Clinical Oncology, Head of Cancer Management and Research department, Medical Research Institute; oral communication, 16 October 2016).

The potential for adverse health effects from occupational exposure to ADs has not been taken seriously by most hospital managers, clinical pharmacists, and nurses themselves. This could be attributed to lack of essential information regarding magnitude of this problem.
Moreover, the implementation of exposure control methods has not been evaluated.

The current study was conducted to determine the potential adverse health effects associated with occupational exposure to ADs and assess adherence to exposure control methods.

\section{MATERIALS AND METHODS}

\section{Study design}

A comparative cross-sectional approach was adopted. ADs-exposed nurses and clinical pharmacists were compared with another group of nonexposed nurses and physicians.

\section{Study setting}

The study was conducted at oncology centers that provide both $\mathrm{ADs}$ preparation and $\mathrm{ADs}$ administration services in Alexandria city and agreed to participate in the research. Those were (a) Oncology Medicine Department at the Alexandria Faculty of Medicine, (b) Cancer Management and Research Department at the Medical Research Institute, and (c) one non-Governmental Oncology Center.

\section{Sampling}

All ADs-exposed nurses and clinical pharmacists, who were registered and practicing during the fieldwork period of the study, were invited to participate $(n=72)$. Initially, 63 responded and had willingness to participate $(87.5 \%)$. However, four nurses and two clinical pharmacists were excluded because their profession duration/occupational history was less than 2 years at the time of the study. Moreover, two supervisory nurses were excluded because they were not involved in mixing and/or administration of ADs. Those who were included in the research $(n=55)$ represented $76.3 \%$ of the overall number of oncology nurses and clinical pharmacists. Another group of nonexposed nurses and physicians who worked at surgical, internal medicine, and cardiology departments at the University Hospitals was included $(n=54)$. ADs-exposed group and nonexposed group were matched regarding their sex, age, marital status, and profession duration.

\section{Study tools}

All participants were subjected to the following research tools: an interview questionnaire.

A structured interview questionnaire was used to collect information regarding the following:

(a) sociodemographic data and occupational history including profession duration.

(b) self-reported adverse reproductive outcomes including impaired fertility (inability to conceive for $>12$ months) and history of spontaneous abortions (unintentional expulsion of an embryo or fetus before the 20th week of gestation).

(c) self-reported hair loss, where the severity of hair loss 
was graded as minimal, moderate, or severe according to pull test, which entails gentle traction on a group of hairs from proximal to distal end. Hairs coming out with each pull were counted. If less than $10 \%$ of pulled hairs come out, is considered as minimal hair loss, up to $20 \%$ is moderate hair loss, and over $30 \%$ is severe hair loss ${ }^{[30]}$.

(d) frequency of oral ulcers were self-reported as none, occasional (1-4/year), or frequent ( $\geq 5 /$ year).

(e) self-reported use of engineering, administrative, and work practice control measures and PPE usage.

\section{Collection of blood samples to evaluate organ toxicity}

Blood samples were collected to conduct the following laboratory investigations:

(a) complete blood count ${ }^{[31]}$ for evaluation of WBC count and differential, platelet count, RBC count, and RBC indices including hemoglobin, hematocrit, mean corpuscular volume $(\mathrm{MCV})$, mean corpuscular hemoglobin $(\mathrm{MCH})$, mean corpuscular hemoglobin concentration (MCHC), and red cell distribution width. For each participant, the value of each parameter was compared with reference range calculated according to age and sex; any value outside the reference range was considered as an abnormal value.

(b) Liver function tests were evaluated by measuring serum alanine transaminase (ALT) and serum aspartate transaminase (AST) levels ${ }^{[32]}$. The reference values of female were 5-33 and 5-32 U/1 for ALT and AST, respectively. The reference values of male were 5-41 and 5-40 U/1 for ALT and AST, respectively.

(c) Kidney function tests were evaluated by measuring the level of serum creatinine (CRE) and blood urea nitrogen $(\mathrm{BUN}){ }^{[33]}$. The normal ranges were 7-23.4 and 0.25-1.20 $\mathrm{mg} / \mathrm{dl}$, respectively. Any biochemical parameter outside the normal range was considered as a positive sign of organ toxicity. Blood samples were analyzed at the Central Laboratories at the Alexandria Faculty of Medicine. Data were collected from the beginning of August 2016 to the end of December 2016.

\section{Statistical analysis of the data}

The collected data were coded, typed onto computer files, tabulated, and analyzed using SPSS software program version 20 (Released 2011, IBM SPSS Statistics for Mac; IBM Corp., Armonk, New York, USA ${ }^{[34]}$. Descriptive statistics included frequency, percentages, mean, and SD. Analytic statistics including parametric and nonparametric tests: Student's t-test and Mann-Whitney test for quantitative variables, and $\chi 2$-test, Fisher's exact test, and Monte Carlo test for qualitative variables were conducted to reveal statistically significant differences between ADsexposed group and nonexposed group regarding selfreported clinical manifestations, and results of laboratory investigations. In addition, a comparison between ADsexposed oncology nurses $(n=37)$ and clinical pharmacists $(n=18)$ regarding the use of engineering, administrative, work practice controls, and PPE usage was done. For all analyses, the level of significance was considered at $5 \%$ $(\alpha=0.05)$.

\section{Ethical considerations}

The study was approved by the Research Ethics Committee of Faculty of Medicine, Alexandria University. An informed written consent was obtained from each participant at the beginning of the study after explanation of the objectives of the study, procedures, and types of information to be obtained. Moreover, participants were aware that they could withdraw from the study at any time without penalty. Collected data were kept confidential.

\section{RESULTS}

The age of ADs-exposed group ranged from 19 to 57 years, with mean age of $35.47 \pm 10.68$ years. Most were females $(92.7 \%)$, and $56.4 \%$ were married. Most of the participants ADs-exposed group $(\mathrm{n}=55)$ comprised 37 $(67.3 \%)$ nurses and $18(32.7 \%)$ clinical pharmacists. Their profession duration ranged from 2 to 34 years, with mean value of $9.5 \pm 8.9$ years (Table 1$)$.

Table 1: Sociodemographic and occupational characteristics of antineoplastic drugs-exposed nurses and clinical pharmacists at oncology centers in Alexandria, 2016

\begin{tabular}{|c|c|c|c|}
\hline $\begin{array}{l}\text { Sociodemographic and occupational } \\
\text { characteristics }\end{array}$ & $\begin{array}{l}\text { Nonexposed group }(\mathrm{n}=54) \\
{[\mathrm{n}(\%)]}\end{array}$ & $\begin{array}{l}\text { ADs-exposed group }(\mathrm{n}=55) \\
{[\mathrm{n}(\%)]}\end{array}$ & Test of significance ( $P$ value) \\
\hline \multicolumn{4}{|l|}{ Sex } \\
\hline Male & $1(1.9)$ & $4(7.3)$ & ${ }^{\mathrm{FE}} P=0.36$ \\
\hline Female & $53(98.1)$ & $51(92.7)$ & \\
\hline \multicolumn{4}{|l|}{ Age } \\
\hline Minimum-maximum & $22-59$ & $19-57$ & $t=-0.066(0.50)$ \\
\hline Mean \pm SD & $34.0 .3 \pm 11.81$ & $35.47 \pm 10.68$ & \\
\hline \multicolumn{4}{|l|}{ Marital status } \\
\hline Single & $27(50)$ & $24(43.6)$ & $\chi^{2}=0.44(0.50)$ \\
\hline Married & $27(50)$ & $31(56.4)$ & \\
\hline
\end{tabular}




\begin{tabular}{|c|c|c|c|}
\hline \multicolumn{4}{|l|}{ Occupation } \\
\hline Nurse & $34(63)$ & $37(67.3)$ & $\chi^{2}=0.22(0.63)$ \\
\hline Clinical pharmacist $/$ physician ${ }^{\mathrm{b}}$ & $20(37)$ & $18(32.7)$ & \\
\hline \multicolumn{4}{|l|}{ Department } \\
\hline Oncology & - & $55(100)$ & \\
\hline Internal Medicine & $17(31.5)$ & - & \\
\hline Surgery & $14(25.9)$ & - & \\
\hline Cardiology & $23(42.6)$ & - & \\
\hline \multicolumn{4}{|l|}{ Profession duration } \\
\hline Minimum-maximum & $2-40$ & $2-34$ & ${ }^{\mathrm{Mw}} P=0.06$ \\
\hline Mean \pm SD & $13.55 \pm 11.40$ & $9.5 \pm 8.90$ & \\
\hline
\end{tabular}

ADs: Antineoplastic drugs, FE: Fisher's exact test, MW: Mann-Whitney test, $\chi 2$ : Chi square test; t: student t-test

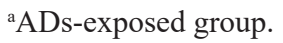

${ }^{b}$ Nonexposed group

Clinical manifestations, as reported by both groups, are presented in Table 2. Married females in ADsexposed group $(\mathrm{n}=29)$ and nonexposed group $(\mathrm{n}=26)$ were asked to report adverse reproductive outcomes. The rate of impaired fertility was significantly higher among ADs-exposed group (31\%) compared with nonexposed group (3.8) ( $\left.{ }^{\mathrm{FE}} P=0.01\right)$. On the contrary, no significant difference was found between both groups regarding number of spontaneous abortions and preterm pregnancies.

No significant difference was found regarding severity of hair loss. However, 23.6 and $12.7 \%$ of ADs-exposed group reported occasional and frequent occurrence of oral ulcers, respectively, compared with $7.4 \%$, and $0.00 \%$ of nonexposed group, respectively; the difference was statistically significant $\left({ }^{\mathrm{MC}} P=0.00\right.$; Table 2).

Table 2: Self-reported adverse reproductive outcomes, hair loss, and occurrence of oral ulcers among antineoplastic drugs-exposed nurses and clinical pharmacists at oncology centers in Alexandria, 2016

\begin{tabular}{|c|c|c|c|}
\hline Clinical manifestations & Nonexposed group [n (\%)] & ADs-exposed group [n (\%)] & Test of significance ( $P$ value) \\
\hline Impaired fertility & $\mathrm{n}=26^{\mathrm{a}}$ & $\mathrm{n}=29^{\mathrm{a}}$ & \\
\hline No & $25(96.2)$ & $20(69)$ & ${ }^{\mathrm{FE}} P=0.01 *$ \\
\hline Yes & $1(3.8)$ & $9(31)$ & \\
\hline \multicolumn{4}{|l|}{ Spontaneous abortions } \\
\hline None & $15(57.7)$ & $23(79.3)$ & ${ }^{\mathrm{MC}} P=0.20$ \\
\hline$<3$ times & $10(38.5)$ & $5(17.2)$ & \\
\hline$\geq 3$ times & $1(3.8)$ & $1(3.4)$ & \\
\hline \multicolumn{4}{|l|}{ Preterm pregnancies } \\
\hline None & $25(96.2)$ & $25(86.2)$ & ${ }^{\mathrm{MC}} P=0.49$ \\
\hline Once & $1(3.8)$ & $2(6.9)$ & \\
\hline Twice & $0(0)$ & $2(6.9)$ & \\
\hline Severity of hair loss & $\mathrm{n}=54$ & $\mathrm{n}=55$ & \\
\hline Minimal (-) & $24(44.4)$ & $15(27.3)$ & $\chi 2=4.17(0.12)$ \\
\hline Moderate (+) & $15(27.8)$ & $16(29.1)$ & \\
\hline Sever $(++)$ & $15(27.8)$ & $24(43.6)$ & \\
\hline
\end{tabular}




\begin{tabular}{|c|c|c|c|}
\hline Occurrence of oral ulcers & $\mathrm{n}=54$ & $\mathrm{n}=55$ & \\
\hline None (-) & $50(92.6)$ & $35(63.6)$ & ${ }^{\mathrm{MC}} P=0.00^{* *}$ \\
\hline Occasional $(+)$ & $4(7.4)$ & $13(23.6)$ & \\
\hline Frequent $(++)$ & $0(0)$ & $7(12.7)$ & \\
\hline
\end{tabular}

ADs, antineoplastic drugs, FE: Fisher's exact test, MC: Monte Carlo test, $\chi 2$ : Chi square test

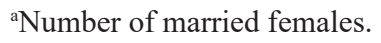

*Significant at $P \leq 0.05$ (two-tailed).

** Significant at $P \leq 0.01$ (two-tailed).

\section{Complete blood count}

The mean WBCs count was significantly lower among ADs-exposed group $(6518 \pm 2064.79 / \mu \mathrm{l})$ compared with nonexposed group $(7307 \pm 2001.40 / \mu \mathrm{l} \mathrm{P}=0.04)$. On the contrary, there was no statistically significant difference between both groups regarding mean RBCs count, mean platelets count, and presence of abnormalities in blood cell counts (Table 3 ).

Table 3: Blood cell count among antineoplastic drugs-exposed nurses and clinical pharmacists at oncology centers in Alexandria, 2016

\begin{tabular}{|c|c|c|c|}
\hline Blood cell count & Nonexposed group $(\mathrm{n}=54)$ & ADs-exposed group $(\mathrm{n}=55)$ & Test of significance ( $P$ value) \\
\hline \multicolumn{4}{|l|}{ RBCs count $\left(10^{6} / \mu \mathrm{l}\right)$} \\
\hline Minimum-maximum & $4.00-5.54$ & $3.66-5.62$ & $t=-0.08(0.93)$ \\
\hline Mean \pm SD & $4.61 \pm 0.31$ & $4.64 \pm 0.42$ & \\
\hline \multicolumn{4}{|l|}{ WBCs count (per $\mu \mathrm{l})$} \\
\hline Minimum-maximum & $4300-13000$ & $2800-12900$ & $t=2.02(0.04)^{*}$ \\
\hline Mean \pm SD & $7307 \pm 2001.40$ & $6518 \pm 2064.79$ & \\
\hline \multicolumn{4}{|l|}{ Platelets count (per $\mu \mathrm{l}$ ) } \\
\hline Minimum-maximum & $109000-429000$ & $177000-485000$ & ${ }^{\mathrm{Mw}} P=(0.11)$ \\
\hline Mean \pm SD & $2946.11 \pm 59145.6$ & $286333.33 \pm 61017.78$ & \\
\hline \multicolumn{4}{|c|}{ Abnormalities in blood cell count } \\
\hline \multicolumn{4}{|c|}{$\mathrm{RBCs}$} \\
\hline Normal & $45(83.3)$ & $47(85.5)$ & ${ }^{\mathrm{MC}} P=0.69$ \\
\hline Abnormal (low) & $0(0)$ & $1(1.8)$ & \\
\hline Abnormal (high) & $9(16.7)$ & $7(12.7)$ & \\
\hline \multicolumn{4}{|l|}{ WBCs } \\
\hline Normal & $50(92.6)$ & $50(90.9)$ & $\chi 2=5.79(0.05)$ \\
\hline Abnormal (low) & $0(0)$ & $4(7.3)$ & \\
\hline Abnormal (high) & $4(7.4)$ & $1(1.8)$ & \\
\hline \multicolumn{4}{|l|}{ Platelets } \\
\hline Normal & $53(98.1)$ & $53(96.4)$ & ${ }^{M C} \mathrm{P}=0.49$ \\
\hline Abnormal (low) & $1(1.9)$ & $0(0)$ & \\
\hline Abnormal (high) & $0(0)$ & $2(3.6)$ & \\
\hline
\end{tabular}

ADs: antineoplastic drugs, MC: Monte Carlo test, MW: Mann-Whitney test, $\chi 2$ : Chi square test, RBCs: red blood cells, WBCs: white blood cells.

*Significant at $P \leq 0.05$ (2-tailed) 
Moreover, the mean $\mathrm{MCH}$ and $\mathrm{MCHC}$ were significantly lower among ADs-exposed group $(26.38 \pm 2.79 \mathrm{fl}$ and $32.08 \pm 1.21 \mathrm{~g} / \mathrm{dl}$, respectively $)$ compared with nonexposed group $(27.24 \pm 2.07 \mathrm{fl}$ and
$32.65 \pm 0.88 \mathrm{~g} / \mathrm{dl}$, respectively) ( ${ }^{\mathrm{MW}} P=0.03$; and $\mathrm{t}=2.77$, $P=0.00$, respectively). No statistically significant difference was found between both groups regarding other RBC indices (Table 4).

Table 4: Red blood cell indices among antineoplastic drugs-exposed nurses and clinical pharmacists at oncology centers in Alexandria, 2016

\begin{tabular}{|c|c|c|c|}
\hline $\mathrm{RBC}$ indices & Nonexposed group $(\mathrm{n}=54)$ & ADs-exposed group $(\mathrm{n}=55)$ & Test of significance ( $P$ value) \\
\hline \multicolumn{4}{|l|}{ Hemoglobin (g/dl) } \\
\hline Minimum-maximum & $10.00-15.40$ & $8.20-16.60$ & $t=1.58(0.11)$ \\
\hline Mean \pm SD & $12.56 \pm 1.08$ & $12.21 \pm 1.46$ & \\
\hline \multicolumn{4}{|l|}{ Hematocrit (PCV) (\%) } \\
\hline Minimum-maximum & $31.00-46.30$ & $10.10-48.50$ & ${ }^{\mathrm{Mw}} P=0.22$ \\
\hline Mean \pm SD & $38.47 \pm 3.07$ & $37.47 \pm 5.55$ & \\
\hline \multicolumn{4}{|l|}{$\operatorname{MCV}(\mathrm{fl})$} \\
\hline Minimum-maximum & $64.70-97.60$ & $59.50-92.70$ & $t=1.17(0.24)$ \\
\hline Mean \pm SD & $83.43 \pm 6.06$ & $82.10 \pm 6.73$ & \\
\hline \multicolumn{4}{|l|}{$\mathrm{MCH}(\mathrm{pg})$} \\
\hline Minimum-maximum & $19.50-30.20$ & $17.40-31.10$ & ${ }^{\mathrm{Mw}} P=0.03 *$ \\
\hline Mean \pm SD & $27.24 \pm 2.07$ & $26.38 \pm 2.79$ & \\
\hline \multicolumn{4}{|l|}{$\mathrm{MCHC}(\mathrm{g} / \mathrm{dl})$} \\
\hline Minimum-maximum & $30.10-34.30$ & $29.20-34.20$ & $t=2.77(0.00)^{* *}$ \\
\hline Mean \pm SD & $32.65 \pm 0.88$ & $32.08 \pm 1.21$ & \\
\hline \multicolumn{4}{|l|}{ RDW $(\%)$} \\
\hline Minimum-maximum & $12.7-17.30$ & $12.80-19.20$ & ${ }^{\mathrm{Mw}} P=0.71$ \\
\hline Mean \pm SD & $14.24 \pm 0.89$ & $14.50 \pm 1.31$ & \\
\hline
\end{tabular}

ADs: antineoplastic drugs, MCH: mean corpuscular hemoglobin, MCHC: mean corpuscular hemoglobin concentration, MCV: mean corpuscular volume, MW: Mann-Whitney test, t: student t-test, RBC: red blood cell, RDW: red cell distribution width.

*Significant at $P \leq 0.05$ (two-tailed).

** Significant at $P \leq 0.01$ (two-tailed).

\section{Liver and kidney function tests}

The mean CRE level was significantly higher among ADs-exposed group $(0.56 \pm 0.13 \mathrm{mg} / \mathrm{dl})$ compared with nonexposed group $\left(0.51 \pm 0.12 \mathrm{mg} / \mathrm{dl}\right.$; $\left.{ }^{\mathrm{MW}} P=0.04\right)$. On the contrary, there was no statistically significant difference between both groups regarding mean ALT, AST, BUN levels, and abnormalities in liver and kidney functions (Table 5).

Table 5: Liver and kidney function tests among antineoplastic drugs-exposed nurses and clinical pharmacists at oncology centers in Alexandria, 2016

\begin{tabular}{|c|c|c|c|}
\hline Liver and kidney FTs & Nonexposed group $(\mathrm{n}=54)$ & ADs-exposed group $(\mathrm{n}=55)$ & Test of significance ( $P$ value) \\
\hline \multicolumn{4}{|l|}{$\operatorname{ALT}(\mathrm{U} / 1)$} \\
\hline Minimum-maximum & $5-63$ & $6-44$ & ${ }^{\mathrm{Mw}} P=0.71$ \\
\hline Mean \pm SD & $15.83 \pm 11.52$ & $14.81 \pm 8.42$ & \\
\hline \multicolumn{4}{|l|}{$\operatorname{AST}(\mathrm{U} / \mathrm{l})$} \\
\hline Minimum-maximum & $11-61$ & $8-53$ & ${ }^{\mathrm{MW}} P=0.14$ \\
\hline Mean \pm SD & $18.90 \pm 7.86$ & $17.62 \pm 7.26$ & \\
\hline
\end{tabular}




\begin{tabular}{|c|c|c|c|}
\hline \multicolumn{4}{|l|}{ BUN (mg/dl) } \\
\hline Minimum-maximum & $6.50-18.70$ & $6.50-19.20$ & $t=-1.54(0.12)$ \\
\hline Mean \pm SD & $10.23 \pm 2.55$ & $11.07 \pm 3.09$ & \\
\hline \multicolumn{4}{|l|}{ CRE (mg/dl) } \\
\hline Minimum-maximum & $0.32-1.03$ & & \\
\hline Mean \pm SD & $0.51 \pm 0.12$ & & \\
\hline \multicolumn{4}{|c|}{ Abnormalities in liver and kidney FTs } \\
\hline \multicolumn{4}{|c|}{ ALT } \\
\hline Normal & $49(90.7)$ & $52(94.5)$ & ${ }^{\mathrm{FE}} P=0.48$ \\
\hline Abnormal (high) & $5(9.3)$ & $3(5.5)$ & \\
\hline \multicolumn{4}{|l|}{ AST } \\
\hline Normal & $52(96.3)$ & $54(98.2)$ & ${ }^{\mathrm{FE}} P=0.61$ \\
\hline Abnormal (high) & $2(3.7)$ & $1(1.8)$ & \\
\hline \multicolumn{4}{|l|}{ BUN } \\
\hline Normal & $53(98.1)$ & $54(98.2)$ & ${ }^{\mathrm{FE}} P=1$ \\
\hline Abnormal (low) & $1(1.9)$ & $1(1.8)$ & \\
\hline \multicolumn{4}{|l|}{ CRE } \\
\hline Normal & $54(100)$ & $55(100)$ & - \\
\hline
\end{tabular}

ADs: antineoplastic drugs, FTs: function tests, ALT: serum alanine transaminase, AST: serum aspartate transaminase, BUN: blood urea nitrogen, CRE: serum creatinine, MW: Mann-Whitney test; FE, Fisher's exact test, t: student t-test

* Significant at $P \leq 0.05$ (two-tailed).

Self-reported use of engineering, administrative, work practice controls, and personal protective equipment by antineoplastic drugs-exposed nurses and clinical pharmacists

Exposure control methods, as reported by the ADsexposed group, are demonstrated in Table 6. Clinical pharmacists were responsible for preparation and constitution of ADs at CAPUs. On the contrary, 13 nurses have been preparing and reconstituting $\mathrm{ADs}$ at openplan treatment areas, and 24 nurses were responsible for administration of ADs to the patients. Biological safety cabinets and ventilation devices were implemented at CAPUs; one biological safety cabinet is present in each unit. Moreover, significantly lower percentage of nurses reported receiving hazard awareness training or ADs safe handling training courses $(13.5 \%)$ compared with the clinical pharmacists $(66.7 \%)(\chi 2=16.02, P=0.00)$. All ADs-exposed group reported lack of medical surveillance program at their workplace.
Regarding adequate safe handling practices, significantly higher percentage of clinical pharmacists (38.9\%) reported storage and transportation of the final product sealed in transport bags compared with nurses $(8.1 \%) \quad\left({ }^{\mathrm{FE}} P=0.01\right)$. Significantly higher percentage of clinical pharmacists $(72.2 \%)$ reported safe disposal of used materials in appropriate containers compared with nurses $(8.1 \%) \quad(\chi 2=24.13, P=0.00)$, and significantly higher percentage of clinical pharmacists $(83.3 \%)$ reported appropriate management of drug spills according to written policies compared with nurses (18.9\%) $(\chi 2=20.93, P=0.00)$. Furthermore, all clinical pharmacists reported wearing single gloves compared with $67.6 \%$ of nurses; besides, most clinical pharmacists reported wearing gowns and surgical masks (94.4 and $72.2 \%$, respectively) compared with nurses ( 8.1 and $8.1 \%$, respectively). The difference between them regarding usage of gloves, gowns, and masks was statistically significant $\left({ }^{\mathrm{FE}} P=0.00 ; \quad \chi 2=39, \quad P=0.00 ; \quad\right.$ and $\chi^{2}=24.13, \quad P=0.00$, respectively; Table 6 ). 
Table 6: Self-reported use of engineering, administrative, work practice controls, and personal protective equipment by antineoplastic drugsexposed oncology nurses and clinical pharmacists at oncology centers in Alexandria 2016

\begin{tabular}{|c|c|c|c|}
\hline Self-reported control methods & $\begin{array}{l}\text { Oncology nurses } \\
\qquad \begin{array}{c}(\mathrm{n}=37) \\
{[\mathrm{n}(\%)]}\end{array}\end{array}$ & $\begin{array}{l}\text { Clinical pharmacists } \\
\qquad(\mathrm{n}=18) \\
{[\mathrm{n}(\%)]}\end{array}$ & $\begin{array}{c}\text { Test of significance } \\
\text { (P value })\end{array}$ \\
\hline \multicolumn{4}{|l|}{ Role at oncology department } \\
\hline Preparation and reconstitution of ADs & $13(35.1)$ & $18(100)$ & $\chi 2=20.71(0.00)^{* *}$ \\
\hline Administration of ADs to patients & $24(64.9)$ & $0(0)$ & \\
\hline \multicolumn{4}{|l|}{ Engineering control measures at workplace } \\
\hline Ventilation device & $0(0)$ & $18(100)$ & ${ }^{\mathrm{FE}} \mathrm{P}=0.00 * *$ \\
\hline Biological safety cabinets & $0(0)$ & $18(100)$ & $\chi 2=55(0.00)^{* *}$ \\
\hline \multicolumn{4}{|l|}{ Administration control measures } \\
\hline Received safety training for adherence to a standard protocol & $5(13.5)$ & $12(66.7)$ & $\chi 2=16.02(0.00)^{* *}$ \\
\hline Medical surveillance program exists at workplace & $0(0)$ & $0(0)$ & \\
\hline \multicolumn{4}{|l|}{ Safe handling practices } \\
\hline Store/transport final product sealed in transport bags & $3(8.1)$ & $7(38.9)$ & ${ }^{\mathrm{FE}} P=0.01 *$ \\
\hline Dispose used materials in appropriate containers & $3(8.1)$ & $13(72.2)$ & $\chi 2=24.13(0.00)^{* *}$ \\
\hline Manage spills according to written policies and procedures & $7(18.9)$ & $15(83.3)$ & $\chi 2=20.93(0.00)^{* *}$ \\
\hline \multicolumn{4}{|l|}{ Use of PPE } \\
\hline Gloves & $25(67.6)$ & $18(100)$ & ${ }^{\mathrm{FE}} P=0.00^{* *}$ \\
\hline Gowns & $3(8.1)$ & $17(94.4)$ & $\chi 2=39(0.00) * *$ \\
\hline Masks & $3(8.1)$ & $13(72.2)$ & $\chi 2=24.13(0.00)^{* *}$ \\
\hline
\end{tabular}

ADs, antineoplastic drugs, FE: Fisher's exact test, $\chi 2$ : Chi square test, PPE: personal protective equipment.

* Significant at $P \leq 0.05$ (two-tailed).

**Significant at $P \leq 0.01$ (two-tailed).

\section{DISCUSSION}

In the present study, ADs-exposed nurses and clinical pharmacists reported significantly higher rate of impaired fertility and oral ulcers. Similarly, Zhang et al. ${ }^{[12]}$, in China, reported that nurses exposed to ADs experience more difficulties in conceiving and have more frequent oral ulcers than nonexposed nurses. In addition, other studies ${ }^{[9,17]}$ revealed that long-term ADs exposure may lead to infertility, and it increases liability to infections.

Regarding hematological parameters in the present study, the mean WBC count, a biomarker for ADs hazard $^{[12]}$, was significantly lower among ADs-exposed nurses and clinical pharmacists compared with nonexposed group. This indicates immune suppression and explains the significantly higher rate of oral ulcers among ADsexposed group. This finding coincides with the result of Zhang et al. ${ }^{[12]}$, in China, where the mean WBC was significantly lower in oncology nurses than in nonexposed nurses. His study also revealed significantly higher number of oncology nurses with abnormal WBC count compared with nonexposed nurses. However, in the current study, there was no significant difference regarding abnormalities in blood cell counts. This could be attributed to a relatively smaller sample size in the present study. Immunological suppression and reduced WBC count owing to ADs exposure were also reported in another study ${ }^{[2]}$.

Regarding RBC indices, few studies examined the association between exposure to ADs and changes in RBCs indices and were conducted among patients with cancer receiving $\mathrm{ADs}^{[35,36]}$. Wenzel et al. ${ }^{[35]}$, showed that exposure to certain ADs may lead to an increase in $\mathrm{MCV}$. Moreover, the European Cancer Anemia Survey ${ }^{[36]}$ reported that $\sim 83 \%$ of patients receiving ADs developed chemotherapy-induced anemia, a hemoglobin level less than $12.0 \mathrm{~g} / \mathrm{dl}$. In the present study, although no significant difference in hemoglobin level or MCV was found between both groups, yet, significantly lower mean $\mathrm{MCH}$ and $\mathrm{MCHC}$ were reported in the ADs-exposed group compared with nonexposed group. In practice, the values of $\mathrm{MCH}$ and $\mathrm{MCHC}$ are used to explain the etiology of anemias; $\mathrm{MCH}$ quantifies the amount of hemoglobin per red blood cell, whereas MCHC correlates the hemoglobin content with the volume of the cell ${ }^{[37]}$. In literature, chemotherapyinduced anemia is well documented ${ }^{[38]}$; however, whether ADs exposure has an effect on $\mathrm{MCH}$ or $\mathrm{MCHC}$ levels is not well documented.

Regarding liver and kidney toxicity, in the current 
study, the mean CRE level was significantly higher in ADs-exposed group compared with nonexposed group, yet, no significant difference between both groups was found on studying abnormalities in ALT, AST, BUN, and CRE levels. On the contrary, Zhang et al. ${ }^{[12]}$, in China, reported significantly higher incidence of abnormal liver and kidney functions among oncology nurses compared with nonexposed nurses. Similarly, liver and kidney toxicities were reported in another study ${ }^{[2]}$. The varied results could be attributed to difference between studies regarding sample size, profession duration, and sufficiency of workplace control procedures as well as PPE usage. Besides, kidney and liver damage might be influenced by other factors, for example, age, preexisting medical condition such as diabetes or elevated blood pressure, family history of kidney or liver disease, reduced fluid intake, and other dietary factors ${ }^{[39,40]}$.

ADs elimination or substitution by a less toxic substance is not feasible, thus, guidelines specify the following exposure control methods:

(a) engineering controls including biological safety cabinets and closed-system transfer devices.

(b) administrative controls such as hazard awareness training, and medical surveillance.

(c) work practice controls such as cleaning spilled chemicals immediately according to written policies.

(d) PPE usage of mainly chemotherapy-tested gloves, single-use disposable gowns, respirators/masks, and eye protection $^{[25]}$.

In the present study, biological safety cabinets and ventilation device were implemented only at CAPUs, whereas most ADs-exposed group participants (67.2\%) have been preparing ADs in an open-plan treatment area. Only one-third of ADs-exposed group participants have received, before or on-job, specialized training courses to become aware about the hazard and understand safe handling practices. Additionally, all ADs-exposed nurses and clinical pharmacists reported lack of medical surveillance program at their workplace; none of them have conducted medical monitoring. In addition, low percentage adopted safe handling practices at their workplace such as transport of the final product sealed in transport bags, safe disposal, and appropriate management of drug spills (18.2, 29.1, and 40\%, respectively). Furthermore, most reported wearing single gloves $(78.2 \%)$, whereas only 36.4 and $29.1 \%$ reported wearing gowns and surgical masks, respectively. In the current study, although significantly higher percentage of clinical pharmacists were adherent to safe handling guidelines compared with nurses, yet, the overall implementation of exposure control measures was inadequate.

The results of the current study coincides with findings of other studies conducted to evaluate adherence to precautionary guidelines for handling ADs, for example, in the study by Boiano et al. ${ }^{[25]}$, similar precautionary guidelines were deficient; however, the performance of nurses versus clinical pharmacists was different. Nurses in their study had better performance in certain guidelines and low performance in others compared with pharmacy practitioners. On the contrary, nurses in the current study reported low performance/adherence to all control methods compared with clinical pharmacists ${ }^{[25]}$. Another study showed that oncology nurses use gloves, but gown use remains comparatively low ${ }^{[28]}$. Furthermore, a survey conducted on a sample Massachusetts nursing population revealed that only $6 \%$ of nurses had training on safe handling procedures, $56 \%$ indicated no special engineering controls at their workplace, and none was aware of the National Institute for Occupational Safety and Health recommended exposure assessment strategies ${ }^{[29]}$.

\section{LIMITATIONS OF STUDY}

Participation in the current study was completely voluntary. Although response rate was $87.5 \%$, yet, the sample size was relatively small. This is attributed to low overall number of registered oncology nurses and clinical pharmacists at the two oncology departments and the oncology center despite that those are the largest three oncology treatment centers in Alexandria City. The current cross-sectional design revealed possible association between occupational exposure to ADs and adverse outcomes; however, prospective studies are better to examine exposure-effect relationship and avoid recall bias. Moreover, it would be better to consider certain factors such as having diabetes or elevated blood pressure, family history of kidney or liver disease, and dietary factors in the current study, as those factors might influence the results of liver and kidney function tests.

\section{CONCLUSION}

This study highlighted chronic adverse health effects associated with occupational exposure to ADs namely impaired fertility, suppressed immunity manifested by oral ulcers and reduced mean WBCs count, and increased mean CRE level. Moreover, the study revealed inadequate implementation of exposure controls particularly among nurses.

It is recommended to utilize the study findings to raise awareness of ADs-exposed personnel regarding ADs hazards and the importance of PPE usage. Additionally, it is recommended to raise awareness among employers to introduce engineering controls in a large scale, conduct hazard awareness training courses, initiate medical surveillance program, and ensure adherence to safe handling practices. In addition, large cross-sectional surveys on ADs-exposed personnel in developing countries are recommended, and occupational exposure to ADs has 
to be evaluated in future research.

\section{ACKNOWLEDGEMENT}

The author very much appreciates the cooperation of nurses, clinical pharmacists, and physicians who participated in the study. The author would like to thank the laboratory personnel and technicians at the Central Laboratories at the Alexandria Faculty of Medicine who carried out the investigations.

The research was supported by the Alexandria Faculty of Medicine that approved the use of laboratories for the investigations.

\section{CONFLICT OF INTEREST}

There are no conflicts of interest

\section{REFERENCES}

1. Edmunds MW. Antineoplastic medications. Chapter 11. In: Edmunds MW, editor. Introduction to clinical pharmacology. 8th ed. Mosby: Elsevier Inc.; 2016. p. 190-197.

2. National Institute for Occupational Safety and Health (NIOSH). Preventing occupational exposures to antineoplastic and other hazardous drugs in healthcare settings. DHHS (NIOSH). Publication No. 2004165. 2004. Available at: http://www.cdc.gov/niosh/ docs/2004-165/pdfs/2004-165.pdf [Accessed 10 October 2016].

3. Fleury-Souverain S, Nussbaumer S, Mattiuzzo M, Bonnabry P. Determination of the external contamination and cross-contamination by cytotoxic drugs on the surfaces of vials available on the Swiss market. J Oncol Pharm Pract 2014; 20: 100-111.

4. Hedmer $\mathrm{M}$, Tinnerberg $\mathrm{H}$, Axmon A, Jönsson BA. Environmental and biological monitoring of antineoplastic drugs in four workplaces in a Swedish hospital. Int Arch Occup Environ Health 2008; 81: 899-911.

5. Harrison BR, Peters BG, Bing MR. Comparison of surface contamination with cyclophosphamide and fluorouracil using a closed system drug transfer device versus standard preparation techniques. Am J Health Syst Pharm 2006; 63: 1736-1744.

6. Sottani C, Porro B, Imbriani M, Minoia C. Occupational exposure to antineoplastic drugs in four Italian health care settings. Toxicol Lett 2012; 213: 107-115.
7. Turci R, Minoia C, Sottani C, Coghi R, Severi $\mathrm{P}$, Castriotta $\mathrm{C}$, et al. Occupational exposure to antineoplastic drugs in seven Italian hospitals: the effect of quality assurance and adherence to guidelines. J Oncol Pharm Pract 2011; 17: 320-332.

8. Yoshida J, Koda S, Nishida S, Yoshida T, Miyajima $\mathrm{K}$, Kumagai S. Association between occupational exposure levels of antineoplastic drugs and work environment in five hospitals in Japan. J Oncol Pharm Pract 2011; 17: 29-38.

9. Connor TH, DeBord DG, Pretty JR, Oliver MS, Roth TS, Lees PS, et al. Evaluation of antineoplastic drug exposure of health care workers at three universitybased US cancer centers. J Occup Environ Med 2010; 52: 1019-1027.

10. Ratner PA, Spinelli JJ, Beking K, Lorenzi M, Chow $\mathrm{Y}$, Teschke $\mathrm{K}$, et al. Cancer incidence and adverse pregnancy outcome in registered nurses potentially exposed to antineoplastic drugs. BMC Nurs 2010; 9: 15 .

11. Friese CR, Himes-Ferris L, Frasier MN. Structures and processes of care in ambulatory oncology settings and nurse-reported exposure to chemotherapy. BMJ Qual Saf 2011; 21: 753-759.

12. Zhang X, Zheng Q, Lv Y, An M, Zhang Y, Wei Y. Evaluation of adverse health risks associated with antineoplastic drug exposure in nurses at two Chinese hospitals: the effects of implementing a pharmacy intravenous admixture service. Am J Ind Med 2016; 59: 264-273.

13. Anwar WA, Salama SI, El Serafy MM, Hemida SA, Hafez AS. Chromosomal aberrations and micronucleus frequency in nurses occupationally exposed to cytotoxic drugs. Mutagenesis 1994; 9: 315-317.

14. Mahrous HS, Ismail SR, Hashishe MM, Kohail HM. Sister chromatid exchanges and chromosome aberrations in lymphocytes of medical personnel handling cytostatic drugs. J Egypt Public Health Assoc 1998; 73: 297-323.

15. El-Ebiary AA, Abuelfadl AA, Sarhan NI. Evaluation of genotoxicity induced by exposure to antineoplastic drugs in lymphocytes of oncology nurses and pharmacists. J Appl Toxicol 2013; 33: 196-201.

16. Connor TH, Lawson CC, Polovich M, McDiarmid MA. Reproductive health risks associated with occupational exposures to antineoplastic drugs in 
health care settings: a review of the evidence. J Occup Environ Med 2014; 56: 901-910.

17. Fransman W, Roeleveld N, Peelen S, de Kort W, Kromhout H, Heederik D. Nurses with dermal exposure to antineoplastic drugs: reproductive outcomes. Epidemiology 2007; 18: 112-119.

18. McDiarmid MA, Oliver MS, Roth TS, Rogers B, Escalante C. Chromosome 5 and 7 abnormalities in oncology personnel handling anticancer drugs. J Occup Environ Med 2010; 52: 1028-1034.

19. International Agency for Research on Cancer. IARC monographs on the evaluation of the carcinogenic risk of chemicals to humans. Lyon, France: World Health Organization, International Agency for Research on Cancer 2016. Available at: http://www.iarc.fr [Accessed 14 September 2016].

20. Pethran A, Schierl R, Hauff K, Grimm CH, Boos KS, Nowak D. Uptake of antineoplastic agents in pharmacy and hospital personnel. Part I: monitoring of urinary concentrations. Intl Arch Occup Environ Health 2003; 76: 5-10.

21. Occupational Safety and Health Administration (OSHA). Controlling occupational exposure to hazardous drugs. OSHA Technical Manual, TED 1-0.15A, section VI, chapter 2. 1999. Available at: http://www.osha.gov/dts/osta/otm/otm vi/otm vi 2.html [Accessed 21 October 2016].

22. Oncology Nursing Society. Cancer chemotherapy guidelines, modules I-V. Pittsburgh: Oncology Nursing Society; 1988.

23. American Society of Health-System Pharmacists. Technical assistance bulletin on handling cytotoxic drugs in hospitals. American Society of Hospital Pharmacists. Am J Hosp Pharm 1985; 42: 131-137.

24. Callahan A, Ames NJ, Manning ML, TouchtonLeonard K, Yang L, Wallen GR. Factors influencing nurses' use of hazardous drug safe handling precautions. Oncol Nurs Forum. 2016; 43: 342-349.

25. Boiano JM, Steege AL, Sweeney MH. Adherence to precautionary guidelines for compounding antineoplastic drugs: a survey of nurses and pharmacy practitioners. J Occup Environ Hyg 2015; 12:588-602.

26. Polovich M, Clark PC. Factors influencing oncology nurses' use of hazardous drug safe handling precautions. Oncol Nurs Forum 2012; 39:E299-E309.

27. Friese CR, Himes-Ferris L, Frasier MN, McCullagh
MC, Griggs JJ. Structures and processes of care in ambulatory oncology settings and nursereported exposure to chemotherapy. BMJ Qual Saf 2012; 21:753-759.

28. Polovich M, Martin S. Nurses' use of hazardous drughandling precautions and awareness of national safety guidelines. Oncol Nurs Forum 2011; 38: 718-726.

29. Fuller TP, Bain EI, Sperrazza K, Mazzuckelli LF. A survey of the status of hazardous drug awareness and control in a sample Massachusetts nursing population. J Occup Environ Hyg 2007; 4: D113-D119.

30. Sperling LC. Evaluation of hair loss. Curr Probl Dermatol 1996; 8: 99-136.

31. Buttarello M, Plebani M. Automated blood cell counts: state of the art. Am J Clin Pathol 2008; 130: 104-116.

32. Pratt DS, Kaplan MM. Laboratory tests. In: Schiff ER, Sorrell MF, Maddrey WC, editors. Schiff's diseases of the liver. 8th ed. Philadelphia: Lippencott-Raven; 1999. 1. p. 205-244.

33. Edmund L, David J. Kidney function tests. In: Carl AB, Edward R, David E, editors. Tietz textbook of clinical chemistry and molecular diagnostics. 4th ed. New Delhi: Elsevier Inc.; 2006. p. 797-808.

34. Kirkpatrick LA, Feeny BC. A simple guide to IBM SPSS statistics for version 20.0. Students ed. Belmont: Wadsworth, Cengage Learning; 2013.

35. Wenzel C, Mader RM, Steger GG, Pluschnig U, Kornek GV, Scheithauer W, et al. Capecitabine treatment results in increased mean corpuscular volume of red blood cells in patients with advanced solid malignancies. Anticancer Drugs 2003; 14: 119-123.

36. Barrett-Lee PJ, Ludwig H, Birgegard G, Bokemeyer C, Gascon P, Kosmidis PA, et al. Independent risk factors for anemia in cancer patients receiving chemotherapy: results from the European cancer anaemia survey. Oncology 2006; 70: 34-48.

37. Bunn HF. Approach to the anemias. Chapter 158. In: Goldman L, Schafer AI, editors. Goldman's cecil medicine. 25th ed. Philadelphia: Elsevier Saunders; 2015. p. 1059-1067.

38. Cortinovis D, Beretta G, Piazza E, Luchena G, Aglione $\mathrm{S}$, Bertolini A, et al. Chemotherapy-induced anemia and oncologist perception on treatment: results of a web-based survey. Tumori 2013; 99: 45-50. 
39. Perazella MA. Renal vulnerability to drug toxicity. Clin J Am Soc Nephrol 2009; 4: 1275-1283.

40. Purkins L, Love ER, Eve MD, Wooldridge CL,
Cowan C, Smart TS, et al. The influence of diet upon liver function tests and serum lipids in healthy male volunteers resident in a Phase I unit. Br J Clin Pharmacol 2004; 57: 199-208. 